\title{
TOMOGRAFIA DE RESISTIVIDAD ELÉCTRICA EN TIEMPO REAL APLICADA A LA INFILTRACIÓN DEL SUELO
}

\section{TIME-LAPSE ELECTRICAL RESISTIVITY TOMOGRAPHY APPLIED TO SOIL INFILTRATION}

\author{
Beatriz Losinno $^{1 *}$, y Claudia Sainato ${ }^{1}$ \\ ${ }^{1}$ Facultad de Agronomía, Universidad de Buenos Aires, Departamento de Ingeniería Agrícola y Uso \\ de la Tierra, Cátedra de Física, Av. San Martín 4453 (C1417DSE), Ciudad de Buenos Aires, Argentina. \\ * Autor para correspondencia E-mail: losinno@agro.uba.ar.
}

\section{RESUMEN}

La infiltración en el suelo juega un papel fundamental en el ámbito agropecuario. Las metodologías estándar para determinar tasa de infiltración básica se ven alteradas por la presencia de vías preferenciales de flujo en el suelo. Esta problemática es relevante cuando se trata de establecimientos de ganadería intensiva, donde la carga contaminante de efluentes es alta. El objetivo de este trabajo fue detectar y caracterizar posibles vías preferenciales de flujo en dos situaciones con distinta intensidad de pisoteo animal: en un sector de un lote de pastura, sin presencia de animales (testigo) y en un sector de tránsito. Se aplicó una lámina de riego con trazador (solución salina) durante un cierto intervalo de tiempo y simultáneamente se llevaron a cabo las tomografías de resistividad eléctrica (TRE) a medida que el flujo de agua iba penetrando en el suelo. La distribución de las anomalías de resistividad eléctrica, respecto a la situación inicial antes del riego (sensible al ingreso del agua salina), observadas a distintos tiempos, indicó el avance de la solución a través del espacio poroso. En el sitio testigo el avance del agua se caracterizó por un frente más homogéneo, mientras que en el sitio de tránsito se visualizaron sectores más acotados, con un flujo mayor y más rápido, que podrían haber dado origen a la contaminación del suelo y agua subterránea detectada en estudios anteriores. Se concluye que esta metodología ha brindado resultados promisorios que sugieren la necesidad de profundizar en este tipo de estudios.

Palabras claves: geoeléctrica, trazador, infiltración

\section{ABSTRACT}

Soil infiltration plays a fundamental role in the agricultural field. Standard methodologies for determining basic infiltration rate are altered by the presence of preferential flow paths in the soil. This problem is important when it comes to intensive livestock farms where the pollutant load of effluents is high. The objective of this work was to detect and characterize possible preferential flow pathways in two situations with different degrees of animal trampling intensity: a site with pasture, with no animals present (control); and a transit site, where effluents flow from Corrals that are in a higher topographic situation. An irrigation sheet was applied with saline water during a certain time periods; simultaneously, time-lapse electrical resistivity tomography (TLERT) was used as the water flow penetrated the soil. The distribution of electrical resistivity anomalies with respect to the initial situation before irrigation (sensitive to salt water ingression), observed at different time periods, showed the advance of the solution through the porous space. In site 1 (control), water advance was characterized by a more homogeneous front; in site 2 , channeling was detected with a greater and 
faster flow, which could have given rise to soil and groundwater contamination detected in previous studies. It is concluded that this methodology has promising results that suggest the need to deepen our knowledge in this area.

Key words: geoelectric, tracer, infiltration

\section{INTRODUCCIÓN}

Durante los últimos años la relación de precios entre los productos ganaderos y los agrícolas, favorable a estos últimos, ha desviado grandes superficies de tierras de alta calidad, anteriormente destinadas al engorde a campo de vacunos, para dedicarlos al cultivo de granos, especialmente soja. Esto ha provocado cambios fundamentales en los métodos de engorde, pasando a un sistema de engorde intensivo a corral, con una alta concentración de animales; en promedio de 300 a 500 animales por hectárea según la elección del manejo de producción (Pordomingo, 2013).

Estas elevadas concentraciones de ganado generan diversos problemas ambientales, fundamentalmente debido a la mayor disposición de desechos sólidos y líquidos (Flores et al., 2012); en particular en la Pampa Húmeda caracterizada porlluvias de aproximadamente $1000 \mathrm{~mm}$ por año, convierte a esta actividad en una fuente puntual de contaminación (García et al., 2013). Dicha contaminación afecta tanto al suelo como al agua, ya sea por escurrimiento superficial llegando a los cursos de agua, como por infiltración profunda hasta las napas subterráneas, originando un incremento de concentración de sales (García et al., 2015).

Por otra parte, el pisoteo animal aumenta significativamente la inestabilidad estructural del suelo (Fernández et al., 2015), conduce al aumento de la densidad superficial del suelo, la disminución de la tasa de infiltración, y de los niveles de porosidad (Denoia et al., 2000) generando compactación. Las propiedades físicas modificadas del suelo debido a la compactación pueden alterar la movilidad de los elementos y cambiar los ciclos de nitrógeno y carbono provocando más emisiones de gases de efecto invernadero (Farrakh Nawaz et al., 2013).

Estudios previos de Martínez et al. (2010b), en el mismo establecimiento feedlot de este trabajo, mostraron que en sectores con alta carga animal y por consecuencia elevado nivel de pisoteo, tanto la tasa de infiltración básica (TIB) medida a campo, como la conductividad hidráulica saturada (Ks) medida en laboratorio, presentan valores significativamente menores a los obtenidos en los sectores no utilizados por los animales. No obstante, los elevados contenidos de nitratos y fósforo observados tanto en el suelo como en el agua subterránea hacen suponer la existencia de vías preferenciales de lixiviación (Sainato et al., 2010). Por ello, la evaluación del proceso de infiltración como vía de entrada de contaminantes en el perfil resulta de importancia en la determinación de indicadores de vulnerabilidad a la contaminación de acuíferos.

Sainato et al. (2012) observaron en este establecimiento que la zona de los corrales y de tránsito de los animales presentaba anomalías de conductividad eléctrica en el suelo y en el agua subterránea.

Si bien la recarga de efluentes a través de la zona no saturada se debería producir en los corrales, Martínez et al. (2010a) detectaron alta resistencia a la penetración del suelo y muy baja permeabilidad hidráulica en estos sectores. Por lo cual, se supuso que el ingreso de contaminantes podría producirse a través de vías preferenciales de flujo, especialmente en la zona de tránsito que presentaba grietas en superficie. El flujo preferencial es un fenómeno común en los suelos que comprende todos los mecanismos donde el agua y los solutos se mueven a lo largo de ciertas rutas, mientras pasan por alto una fracción de la matriz porosa (Wehrer y Slater, 2015).

La hipótesis propuesta fue que, en un suelo compactado por el pisoteo animal, pueden existir caminos preferenciales que permitirían la infiltración de contaminantes al agua subterránea.

Se plantea estudiar el movimiento del flujo de agua en suelos de corrales y alrededores de un establecimiento de ganadería intensiva utilizando la tomografía de resistividad eléctrica (TRE) en combinación con lluvia simulada con un trazador.

El objetivo específico de este trabajo consistió en detectar y caracterizar posibles vías preferenciales de flujo, comparando un sitio testigo, representado por un sector no transitado por animales, con el sitio donde el pisoteo animal ha generado compactación.

\section{MATERIALES Y MÉTODOS}

\section{Descripción de la zona de estudio}

El estudio se llevó a cabo en un establecimiento dedicado a la cría y al engorde intensivo de ganado vacuno, ubicado en las proximidades de la localidad de Marcos Paz, provincia de Buenos

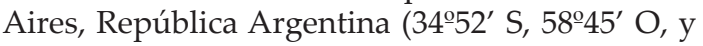
$25 \mathrm{msnm})$. 
La litología de la zona se caracteriza, desde la superficie, por una secuencia sedimentaria de limo y limo arcilloso (Formaciones Platense y Lujanense), sobre otra secuencia de limo y limo arcilloso con costra calcárea (BonaerenseEnsenadense). Esta última contiene la Formación Epipuelches que incluye el acuífero freático y el Pampeano; debajo de éste se ubican las arenas Puelches. Las isolíneas del nivel freático se ubican de unos pocos metros hasta $8 \mathrm{~m}$ de profundidad (Auge, 2004) y son similares a las curvas de nivel topográfico regional.

La dirección del flujo del agua subterránea es aproximadamente SN y presenta dentro del corral una pendiente que disminuye en la dirección SO-NE. Los corrales están en una posición ligeramente más alta que los caminos circundantes (Sainato et al., 2012).

El manejo del establecimiento consistía en corrales de engorde, algunos sectores destinados al pastoreo directo y otros a la producción de forrajes, sin presencia de animales.

Las calicatas realizadas por Martínez et al. (2010a), permitieron clasificar al suelo como un Argiudol de textura Franco limosa, detallado en la Tabla 1. Serie San Vicente (INTA).

Los sitios estudiados 1 y 2 , presentan una situación sin presencia de animales considerada Testigo y otra sometida al pisoteo del ganado, respectivamente.

Las características fisicoquímicas del suelo en los sitios se obtuvieron de Sainato et al. (2012) y se describen en la Tabla 2.

\section{Diseño experimental}

Para este ensayo se eligieron dos sitios, siguiendo un diseño experimental dirigido basado en los ensayos previos realizados en dicho establecimiento (Sainato et al., 2012) que permitió detectar zonas críticas.

El sitio 1 (Testigo) corresponde a un sector del potrero de 3 ha destinado a producción de forraje, con una pastura implantada, sin presencia de animales que se encuentra a $400 \mathrm{~m}$ de los corrales, y en una situación topográfica más baja.

El sitio 2 fue sometido a intenso pisoteo, ubicado en la calle de vinculación de los corrales de engorde (franja de $200 \mathrm{~m}^{2}$ ) contigua a una zanja de efluentes. Este sitio presentaba visualmente pequeñas grietas superficiales y anomalías

Tabla 1. Valores medios (\%) de arcilla, limo y arena entre 0 y $20 \mathrm{~cm}$ de profundidad, correspondiente a los dos sitios estudiados. Contenido de Carbono Total (CoT), densidad aparente (DAp) $\left(\mathrm{g} \mathrm{cm}^{-3}\right)$, Resistencia a la penetración (Rp) (MPa), Conductividad Hidráulica (Ks), Tasa de Infiltración Básica (TiB) en $\mathrm{mm} \mathrm{h}^{-1}$ para dos intensidades de pisoteo. Obtenido de Martinez et al. (2010 a y b)

Table 1. Mean values (\%) of clay, silt and sand between 0 and $20 \mathrm{~cm}$ of depth, corresponding to the two sites studied. Total Carbon Content (CoT), bulk density (DAp) $\left(\mathrm{g} \mathrm{cm}^{-3}\right)$, Penetration Resistance (Rp) (MPa), Hydraulic Conductivity (Ks), Basic Infiltration Rate (TiB) in $\mathrm{mm} \mathrm{h}^{-1}$ for two trampling intensities. Retrieved from Martinez et al. (2010 a and b)

\begin{tabular}{lccccccccc}
\hline Profundidad & Arcilla & Limo & Arena & CoT & DAp & Rp & Ks & TiB & Int. pisoteo \\
\hline 0-20 cm & $\%$ & $\%$ & $\%$ & $\mathrm{~g} \mathrm{~kg}^{-1}$ & $\mathrm{~g} \mathrm{~cm}^{-3}$ & $\mathrm{MPa}$ & $\mathrm{mm} \mathrm{h}^{-1}$ & $\mathrm{~mm} \mathrm{~h}^{-1}$ & \\
Sitio 1 (T) & 27.5 & 50.6 & 21.9 & 52.0 & 1.23 & 4.41 & 37.3 & 3.48 & nulo \\
Sitio 2 & 25.0 & 57.5 & 17.5 & 66.0 & 1.28 & 7.67 & 9.6 & 1.93 & alto \\
\hline
\end{tabular}

T: Testigo

Tabla 2. Propiedades del suelo obtenidas del muestreo realizado por Sainato et al. (2012), profundidad de la muestra, $\mathrm{pH}$, conductividad eléctrica del extracto pasta de saturación (CEs), concentraciones de fósforo (P), carbono oxidable (Cox) y las de $\mathrm{Ca}, \mathrm{Mg}$, $\mathrm{Na}$ y $\mathrm{K}$.

Table 2. Soil properties of the sample made by Sainato et al (2012), depth of the sample, pH, electrical conductivity of the saturation paste extract (CEs), and contents of phosphorus (P), oxidizable carbon (Cox), Ca, Mg, $\mathrm{Na}$ and $\mathrm{K}$.

\begin{tabular}{lllccccccc}
\hline Prof, & pH & CEs & P & NO3 & Cox & k & Na & Mg & Ca \\
\hline 0-20cm & & $\mathrm{S} \mathrm{m}^{-1}$ & $\mathrm{mg} \mathrm{kg}^{-1}$ & $\mathrm{mg} \mathrm{kg}^{-1}$ & $\mathrm{~g} \mathrm{~kg}^{-1}$ & $\mathrm{cmolc} \mathrm{kg}^{-1}$ & $\mathrm{cmolc} \mathrm{kg}^{-1}$ & cmolc kg-1 & $\mathrm{cmolc} \mathrm{kg}^{-1}$ \\
Sitio 1(T) & 6 & 0.02 & 18 & 30 & 12.5 & 1.6 & 0.3 & 1.4 & 8.3 \\
Sitio 2 & 6.9 & 0.2 & 30 & 88 & 24.8 & 2.2 & 1.0 & 3.2 & 8.1 \\
\hline
\end{tabular}

T: Testigo 
de alta conductividad eléctrica en la zona no saturada y altos contenidos de nitratos (Sainato et al., 2012) como se observa en la Tabla 2. Garel et al. (2012) caracterizaron que las macro-fisuras presentes en la superficie pueden actuar como posibles vías de ingreso de agua. Se delimitó una parcela de $6 \mathrm{~m}$ por $6 \mathrm{~m}$ en cada uno de los sitios, donde se colocó un dispositivo para el riego simulado. Se construyó una tubería en forma de U (Fig. 1) en cuyos cuatro vértices se colocaron microaspersores modulares con girador a $90^{\circ}$ y en la rama de la $U$ menos afectada por las turbulencias se hizo una derivación para agregar en el centro del diseño un quinto microaspersor rotatorio (EIN DOR® modelo 866, TAVLIT, Norte Yavne, Israel) que genera aspersión a $360^{\circ}$; esto posibilitaba una distribución uniforme del riego.

Para contemplar los efectos de borde, dentro de dichas parcelas se demarcó una superficie de 4 $\mathrm{m}$ por $4 \mathrm{~m}$, en el que se efectuó riego por aspersión con solución salina $(\mathrm{KBr})$ de concentración $5 \mathrm{~g} \mathrm{~L}^{-1}$ (cuya salinidad es diez veces mayor que la del agua corriente para consumo). Esta sal ha mostrado ser adecuada como trazador, ya que su presencia modifica la resistividad eléctrica del perfil de suelo a medida que va avanzando, sin provocar alteraciones en sus agregados (Domínguez et al., 2011). La solución se almacenó en un tanque de 500 litros, y era impulsada por una bomba periférica (GAMMA mod. QB60®, Grupo Simpa S.A., Parque Industrial Garín, Argentina) con potencia de 0,37 $\mathrm{kW}$. El sistema generaba un caudal de salida de aproximadamente $200 \mathrm{~L} \mathrm{~h}^{-1}$, que para el tiempo de duración del ensayo implicó la aplicación de una lámina de alrededor de $6 \mathrm{~mm}$, lo que coincide aproximadamente con una lluvia leve. La intensidad de la lluvia fue constante durante las mediciones, se midió a través de seis pluviómetros distribuidos por toda la parcela para verificar que la distribución del riego fuera homogénea.

El ensayo consistió en incorporar al suelo una sustancia en forma de solución, que modifique alguna de las propiedades del mismo, en este caso su conductividad eléctrica (CE) a medida que va avanzando a lo largo del perfil.

Por medio de TRE se puede medir la resistividad eléctrica aparente (@a) (inversa de la CEa, conductividad eléctrica aparente) en tiempo real (De Franco et al., 2009).

Los valores de oa en el perfil constituyen la distribución de resistividad aparente en profundidad de los datos observados a partir de un número importante de repeticiones (Oldenburg y Li, 1994).

Para medirla resistividad aparente se realizaron TRE, para lo cual se utilizó un resistivímetro Saris (Scintrex LTd, Ontario, Canadá). Se utilizó la configuración DIPOLO - DIPOLO (Fig. 2), que consiste en hacer circular corriente entre los electrodos de corriente A y B y medir la diferencia de potencial entre los electrodos $\mathbf{M}$ y $\mathbf{N}$ (Telford et al., 1990) obteniéndose la distribución de resistividad eléctrica aparente $\left(\varrho_{\mathrm{a}}\right)$ (Losinno et al., 2008).

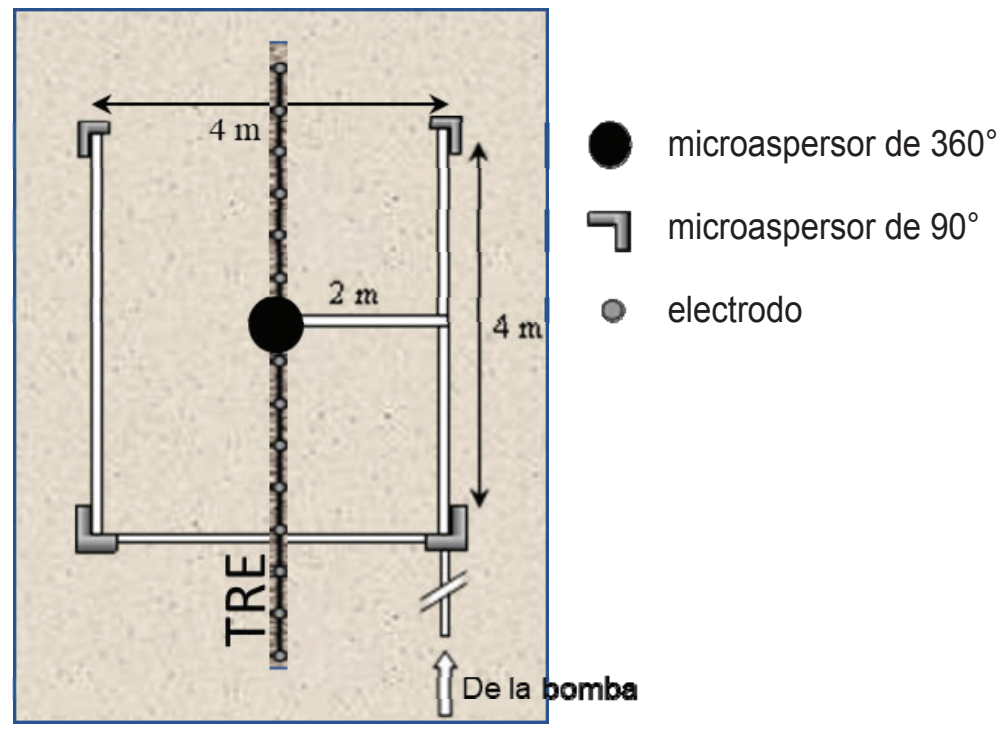

Fig. 1. Vista en planta del sistema de riego y ubicación de la línea de electrodos (TRE). Se muestran los cinco micro-aspersores por parcela.

Fig. 1. Plan view of the irrigation system and location of the electrode line (TRE). The five microsprinklers are shown. 


\section{Configuración de los electrodos del método dipolo-dipolo}

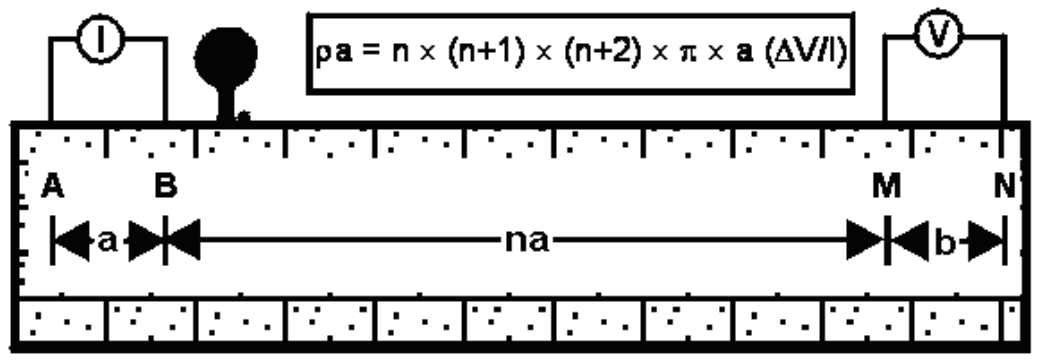

A, B : electrodos de corriente

$\mathbf{M}, \mathbf{N}$ : electrodos de potencial

$\mathrm{a}=\mathrm{b}$ : espaciamiento de los electrodos

de corriente y de potencial respectivarnerte

Modificado según DoBRIN 品 SAVIT, 1988 ieleccc.cdr, S.G rierrkle

Fig. 2. Esquema de la configuración de electrodos dipolo-dipolo utilizada.

Fig. 2. Diagram of the configuration of the dipole-dipole electrodes used.

Los electrodos se conectan mediante cables a una caja de contactos, a través de la cual se seleccionan las distancias entre los electrodos utilizados, se toma de a cuatro electrodos secuencialmente y luego, incrementando la distancia entre electrodos de corriente y los de potencial se van registrando los valores de la oa correspondiente a niveles del subsuelo a mayor profundidad. En este trabajo se armó una transecta de veintiún electrodos de acero inoxidable distanciados a $20 \mathrm{~cm}$, con una apertura máxima de $4 \mathrm{~m}$. Con esta configuración se obtuvieron 50 datos experimentales, que contribuyen a la descripción de la resistividad del volumen de suelo hasta aproximadamente un metro de profundidad, por debajo de cada sitio estudiado.

Las mediciones se realizaron lo suficientemente rápido para detectar el progreso temporal de la infiltración; Zieher et al. (2017) utilizaron intervalos de $7 \mathrm{~min}$, en este trabajo se efectuaron las mediciones entre 15 y $20 \mathrm{~min}$.

Los datos experimentales requieren del proceso matemático de inversión, este paso es esencial para la interpretación de los datos ya que permite obtener un modelo que represente la verdadera distribución de resistividad eléctrica en el suelo (Arora y Ahmed, 2011). Zieher et al. (2017), monitorearon procesos de infiltración a través de TRE en tiempo real y obtuvieron modelos bidimensionales. En nuestro caso, para el procesamiento de los datos experimentales se utilizó el programa de inversión DCIP2D (UBCGIF) (Oldenburg y Li, 1994). El programa provee un modelo bidimensional de resistividades del subsuelo a partir del conjunto de datos experimentales utilizando una rutina de inversión por cuadrados mínimos. Dicho modelo es aquél cuya respuesta de $\varrho_{a}$ es la que mejor ajusta a los datos experimentales, minimizando la función de ajuste definida como:

$$
\phi=\sum_{i=1}^{50}\left(\frac{\rho_{a i}^{\text {predicho }}-\rho_{a i}{ }^{\text {observado }}}{\varepsilon_{i}}\right)^{2}
$$

donde $\varepsilon_{\mathrm{i}}$ es el error experimental.

Se efectuó una TRE inicial, antes de comenzar la infiltración y a continuación, ya con el riego funcionando, se efectuaron cuatro TRE en distintos instantes de tiempos y se obtuvieron los modelos de capas correspondientes.

Para caracterizar la variación de resistividad aparente causado por la presencia de la solución salina que se infiltra en los poros del suelo, se calculó el valor absoluto de la anomalía de resistividad eléctrica para cada tiempo, y la variación de la resistividad eléctrica en porcentaje para cada profundidad (Samouelian et al., 2005). La anomalía se calculó como:

$$
\frac{\rho_{a}^{o}-\rho_{a}^{i}}{\rho_{a}^{o}} \cdot 100 \quad(\text { Ec. } 2)
$$

donde $\rho_{a}^{o}$ y $\rho_{a}^{i}$ son las resistividades eléctricas aparentes a tiempo inicial (antes de la infiltración) $\mathrm{y}$ a un instante $t$ posterior, respectivamente (Travelletti et al., 2012) 


\section{RESULTADOS Y DISCUSIÓN}

Las variaciones en el contenido de agua asociadas con un frente de infiltración dominan la respuesta eléctrica observada durante eventos de infiltración a corto plazo, lo que permite el análisis de la dinámica del agua a partir de los datos de la TRE (Wehrer y Slater, 2015).

Los modelos de resistividad eléctrica aparente $\left(\mathrm{Q}_{\mathrm{a}}\right)$ observada, se muestran en las Figs. 3a y $3 \mathrm{~b}$ para el sitio 1 (testigo) y 2 (canaleta de efluentes), respectivamente. Estas imágenes representan la distribución de la oa del suelo, obtenidas de las TRE en cuatro tiempos posteriores al inicio de la infiltración.

Las zonas con menor valor de resistividad eléctrica en dichas imágenes pueden ser interpretadas como el avance del frente húmedo en el proceso de infiltración a distintos intervalos de tiempo.

En la Fig. 3a el primer gráfico corresponde al estado inicial (antes de la infiltración), en esta instancia el valor de $\mathrm{Q}_{\mathrm{a}}$ obtenido para la situación inicial es de alrededor de 70 ohmm. A continuación, sigue una secuencia a distintos instantes de tiempo, contados a partir del comienzo de la lluvia simulada que se interrumpe a los $78 \mathrm{~min}$. Se observa con línea punteada como va evolucionando el frente salino a través de la disminución de la $Q_{a}$. A partir de los $25 \mathrm{~min}$ se observa un primer avance del agua salina, comenzando en la zona central, extendiéndose paulatinamente hacia los laterales y en profundidad, zona que se va ampliando posteriormente.

La Fig. 3b muestra una situación inicial más heterogénea, donde a ese sitio llega el escurrimiento superficial de efluentes proveniente de los corrales. En consecuencia, el valor de oa de dicho perfil es menor, cercano a los $20 \mathrm{ohm}$ $m$ en valor promedio (antes de la infiltración). Al cabo de 20 min de comenzada la lluvia simulada, se percibe un descenso de oa, en una zona que se perfila como vía preferencial de flujo de la solución; a los 50 min se visualiza una segunda vía y al cabo de $2 \mathrm{~h}$ hay tres zonas asociadas a un descenso de la $Q_{a}$. La lluvia se interrumpe a los $90 \mathrm{~min}$

Flores et al. (2012) detectaron sitios con alto riesgo ambiental relacionado con el aumento del número del ganado confinado, impactando en las áreas que rodean los recursos hídricos.

Las Figs. $4 \mathrm{a}$ y $4 \mathrm{~b}$ presentan, en valor absoluto, los porcentajes de las anomalías de resistividad

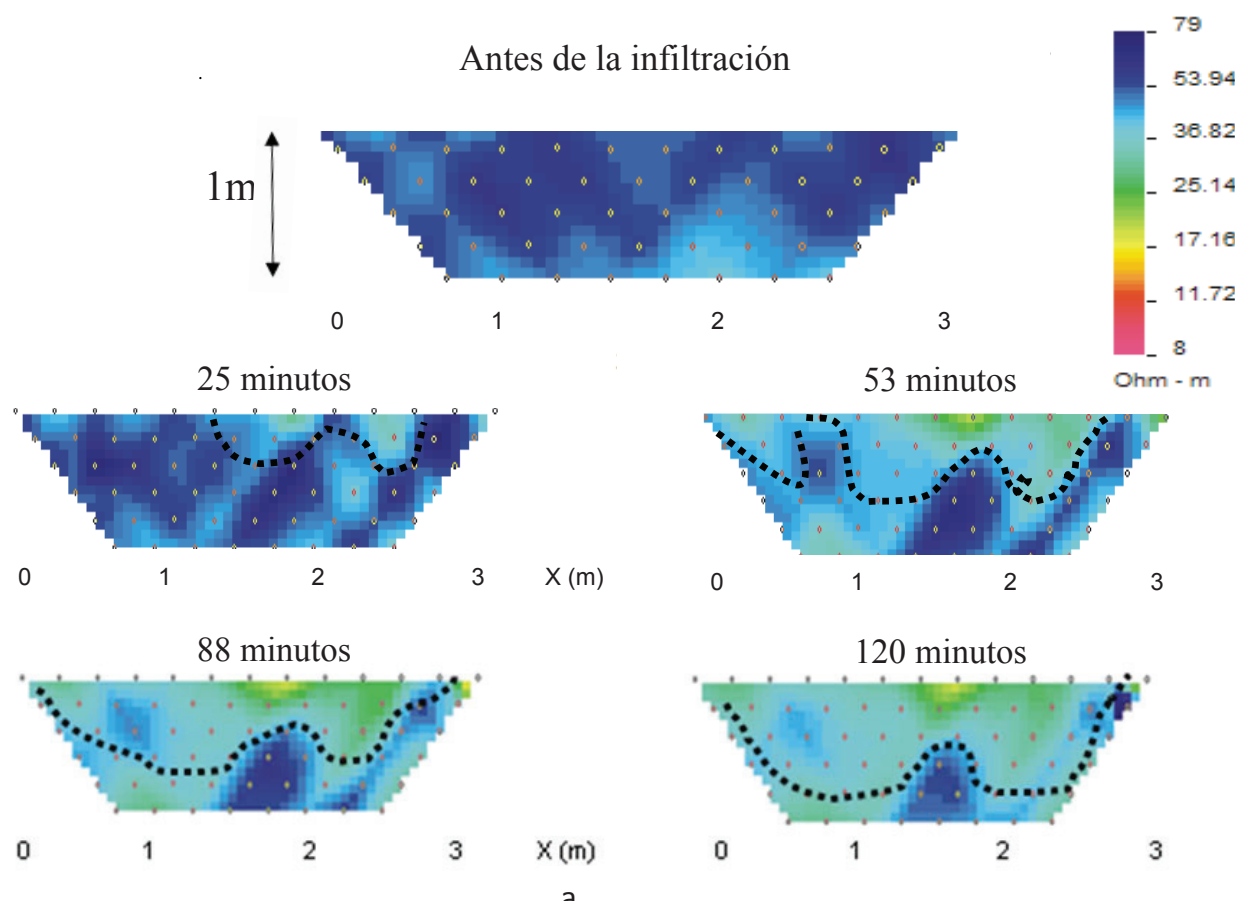

Fig. 3a. Resistividad aparente observada. Sitio 1 - Testigo. Situación inicial y evolución del frente salino a distintos tiempos; el riego se suspendió a los $78 \mathrm{~min}$. La línea punteada indica la evolución del frente.

Fig. 3a. Observed apparent resistivity. Site 1 - Control. Initial situation and evolution of the saline front at different time periods; irrigation was stopped after 78 minutes. The dotted line indicates the evolution of the saline front. 


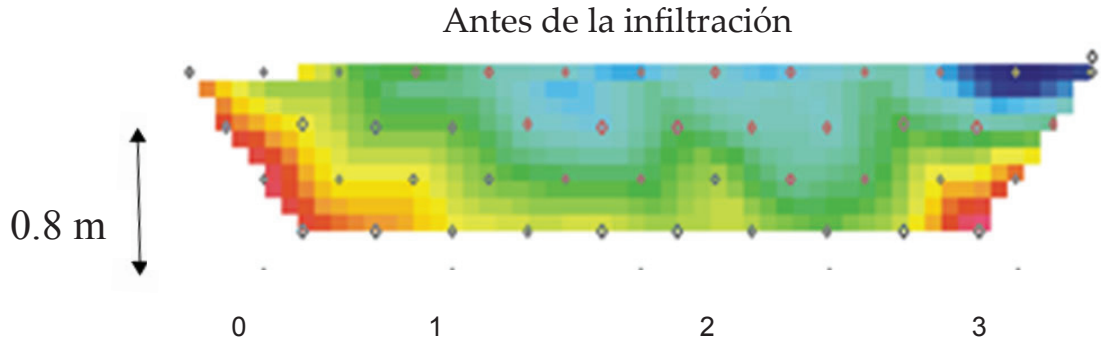

20 minutos

50 minutos

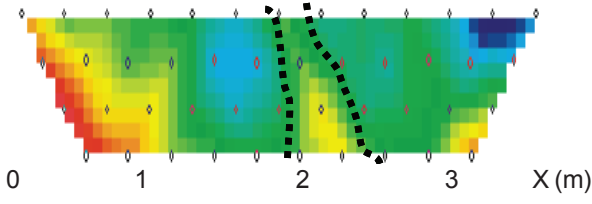

95 minutos

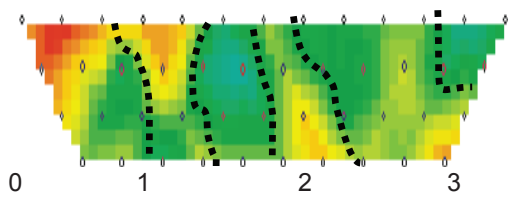

$\mathrm{X}(\mathrm{m})$

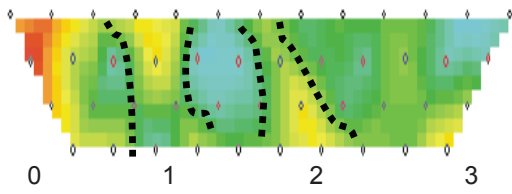

123 minutos

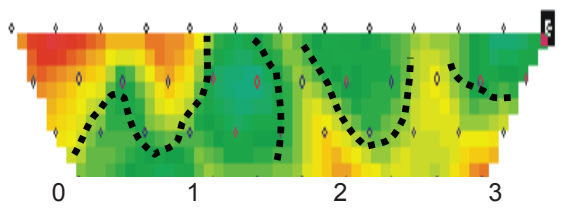

\section{b}

Fig. 3b. Resistividad aparente observada. Sitio 2- Canaleta de efluentes. Situación inicial y evolución del frente salino a distintos tiempos; el riego se suspendió a los 90 minutos. La línea punteada señala el avance de la solución salina.

Fig. 3b. Observed apparent resistivity. Site 2-Effluent channel. Initial situation and evolution of the saline front at different time periods; irrigation was stopped after $\mathbf{9 0}$ minutes. The dotted line indicates the evolution of the saline front.

eléctrica en diferentes momentos de la infiltración en cada sitio respectivamente.

En el caso del sitio 1 (Fig. 4a) la anomalía es casi nula en la primera etapa, empezando sucesivamente a aumentar en las mismas zonas señaladas en la Fig. 3a, indicando la llegada del frente salino. El valor máximo de la anomalía en la situación final fue de un $75 \%$ aproximadamente en una zona pequeña localizada en el centro del sondeo, predominando anomalías del orden del $35 \%$ en el resto del perfil. Este resultado coincide con el de Arora y Ahmed (2011), quienes hallaron que los cambios en la resistividad en la zona vadosa, debido a la infiltración eran del orden del $25 \%$.

Para el sitio 2 (Fig. 4b) recién en la tercera etapa se visualizan anomalías (en valor absoluto) en una zona muy limitada, del $25 \%$, coincidiendo con vías de infiltración señaladas en la Fig. 3b. Los contrastes de las anomalías son menores en el caso de la canaleta ya que la situación inicial del suelo, debido a la alta salinidad generada por los efluentes que presentaba, correspondía a valores bajos de $\mathrm{Q}$ (Sainato et al., 2012).

La Fig. 5a, muestra para seis profundidades en el sitio 1, las variaciones de la resistividad eléctrica medida en porcentaje. Para cada instante de tiempo se tomó la diferencia entre el valor de resistividad del modelo inicial y el medido en ese tiempo, obtenidos de los modelos de las TRE. Superficialmente la variación de resistividad es positiva, determinando que efectivamente al transcurrir el tiempo aumenta la CE (inversa de la resistividad), debido a la presencia del trazador; a partir de los $80 \mathrm{~cm}$ se visualiza una pequeña zona, que se va agrandando en profundidad, que pareciera originalmente menos resistiva (variación negativa) coincidente con el modelo.

La Fig. $5 b$ muestra para cuatro profundidades las variaciones de la resistividad eléctrica medida en porcentaje para el sitio 2 . A diferencia del sitio anterior, en superficie $(20 \mathrm{~cm})$ las variaciones de resistividad son negativas durante todo el periodo de tiempo ensayado, evidenciando la presencia de una capa más conductora inicial, debido a la presencia salina de los efluentes vertidos en dicha canaleta, que se va disipando en profundidad y revirtiendo esta tendencia.

Se visualizan, a partir de los $40 \mathrm{~cm}$, máximos de variaciones positivas; manifestando el ingreso 
0
50
100

$\mathrm{cm}$

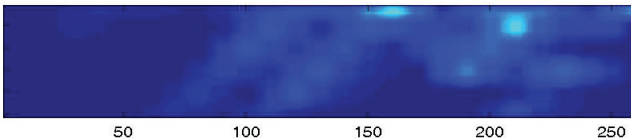

100

150

200

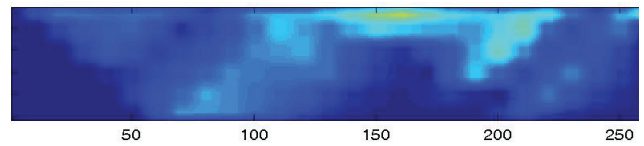

50

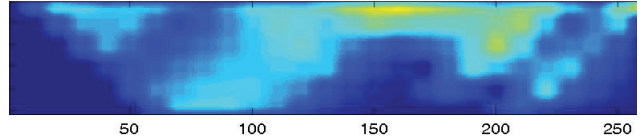

50

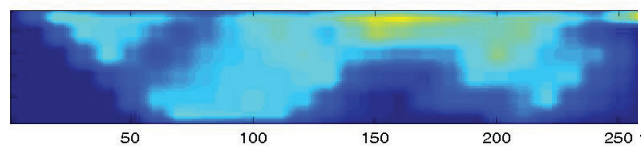

a
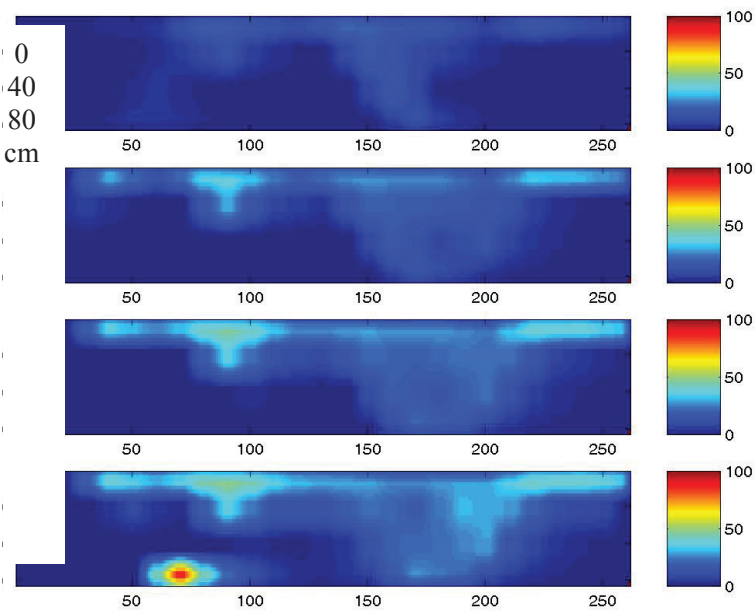

b

Fig. 4a. Sitio 1. Porcentajes de anomalías de resistividad (en modulo) a los instantes de tiempos descriptos: $\mathrm{T} 1=25 \mathrm{~min}, \mathrm{~T} 2=53 \mathrm{~min}, \mathrm{~T} 3=88 \mathrm{~min}, \mathrm{~T} 4=120 \mathrm{~min}$.

Fig. 4a. Site 1. Percentages of resistivity anomalies (in module) at the time periods described: T1 = 25 $\min , \mathrm{T} 2=53 \mathrm{~min}, \mathrm{~T} 3=88 \mathrm{~min}, \mathrm{~T} 4=120 \mathrm{~min}$.

Fig. 4b. Sitio 2. Porcentajes de anomalías (en módulo) de resistividad a los intervalos de tiempos descriptos: $\mathrm{T} 1=20 \mathrm{~min}, \mathrm{~T} 2=50 \mathrm{~min}, \mathrm{~T} 3=95 \mathrm{~min}, \mathrm{~T} 4=123 \mathrm{~min}$.

Fig. 4b. Site 2. Percentages of resistivity anomalies (in module) at the time periods described: T1 $=20$ $\min , \mathrm{T} 2=50 \mathrm{~min}, \mathrm{~T} 3=95 \mathrm{~min}, \mathrm{~T} 4=123 \mathrm{~min}$.

del trazador en zonas puntuales (de mayor resistividad inicial) coincidente con las vías de infiltración (Fig. 3a).

Si bien los valores de tasa de infiltración básica en este corral son muy bajas, las muestras de suelo y agua subterránea contienen altas concentraciones de nitratos, fósforo y otros iones (Martínez et al., 2010b). Las vías preferenciales de infiltración obtenidas en los resultados de la Fig. 3a, podrían señalar el origen del incremento en estas concentraciones a pesar de las altas resistencias a la penetración medidas, indicador natural de la compactación del suelo.

En las mediciones de TRE en tiempo real que se llevan a cabo a lo largo de varios días o períodos de tiempo más largos, es necesario realizar un registro de la temperatura del suelo (Travelletti et al., 2012). Como la duración del presente experimento de lluvia simulada fue de dos horas, estos efectos no se consideraron (Zieher et al., 2017), por lo tanto, los cambios en la resistividad eléctrica se deben principalmente al cambio en el contenido de agua del suelo y su salinidad. Similares resultados obtuvieron Wehrer y Slater (2015) quienes pudieron caracterizar las curvas de avance del trazador mediante TRE.

\section{CONCLUSIONES}

Se analizó el proceso de infiltración de agua con un trazador a través de tomografía de resistividad eléctrica en dos situaciones diferentes: una pastura y un sitio aledaño a corrales de engorde de vacunos, con alta carga y pisoteo animal. Se verificó que el patrón de distribución del frente húmedo en profundidad en la canaleta de efluentes es más heterogéneo que en el caso del suelo de pastura donde es más uniforme.

Se comprobó que este último sitio, a pesar de la alta compactación del suelo, resultó una fuente puntual de contaminación a través de vías preferenciales de infiltración, detectadas por la presencia de la solución salina en profundidad. Por lo tanto, la zona de descarga de efluentes presenta un riesgo de lixiviación rápida de sustancias contaminantes.

La metodología de monitoreo eléctrico en tiempo real ha brindado resultados promisorios que sugieren la necesidad de profundizar en este tipo de estudios, para identificar parámetros que permitan cuantificar la vulnerabilidad de cada sitio en función de las vías preferenciales de flujo. 

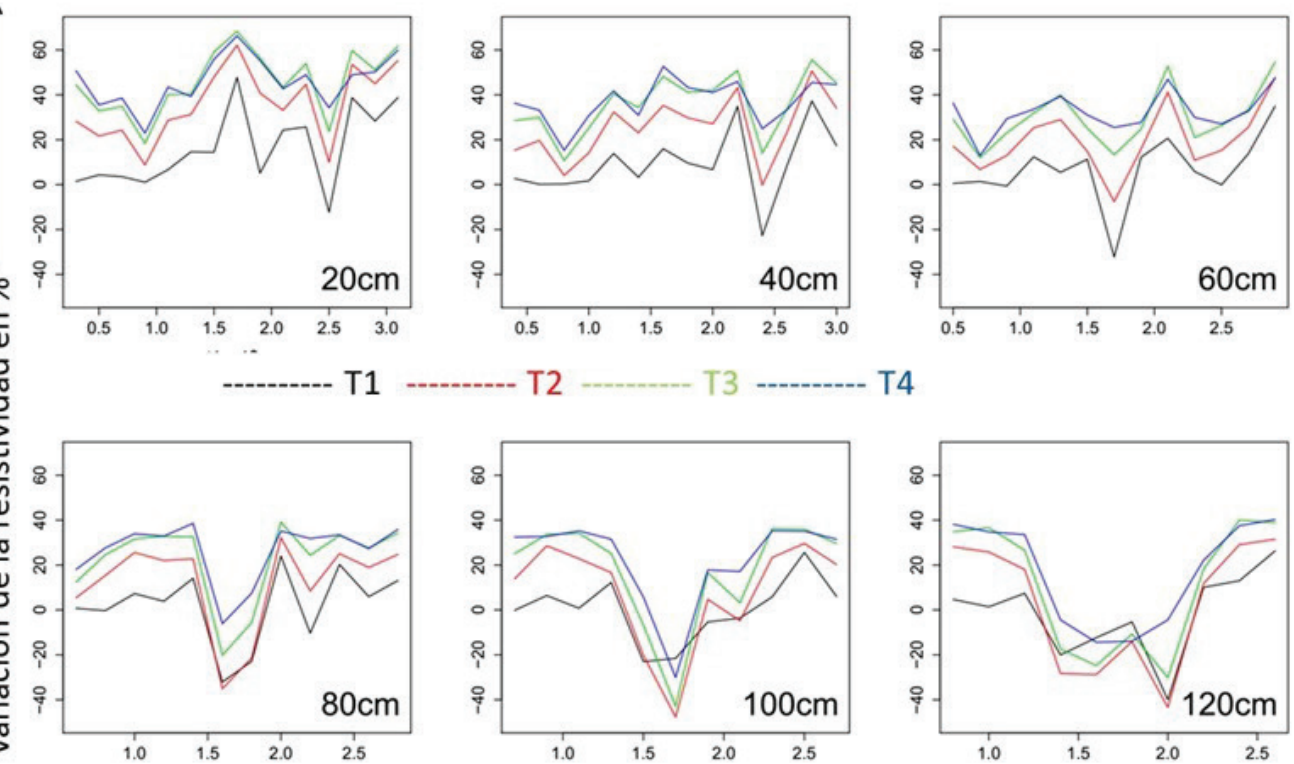
$-\mathrm{T} 2$ T4
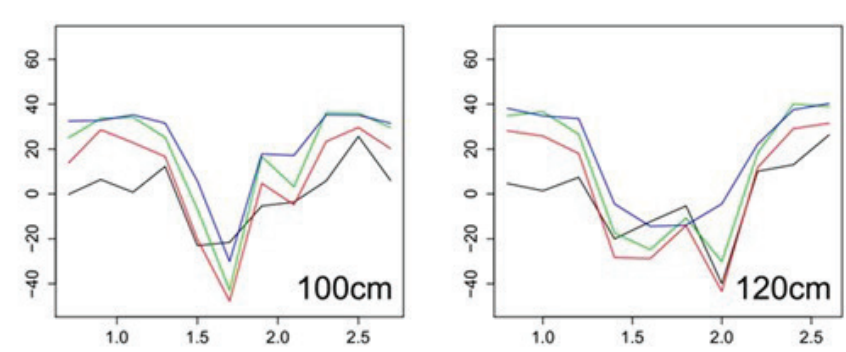

Distancia a lo largo del perfil (m)

Fig. 5a. Variación de la resistividad eléctrica (\%) en diferentes tiempos a lo largo del perfil; a distintas profundidades en el sitio S1.

Fig. 5a. Variation of electrical resistivity (\%) at different time periods along the profile, at different depths in site S1.

$\mathrm{T} 1=25 \mathrm{~min}, \mathrm{~T} 2=53 \mathrm{~min}, \mathrm{~T} 3=88 \mathrm{~min}, \mathrm{~T} 4=\mathbf{1 2 0} \mathrm{min}$
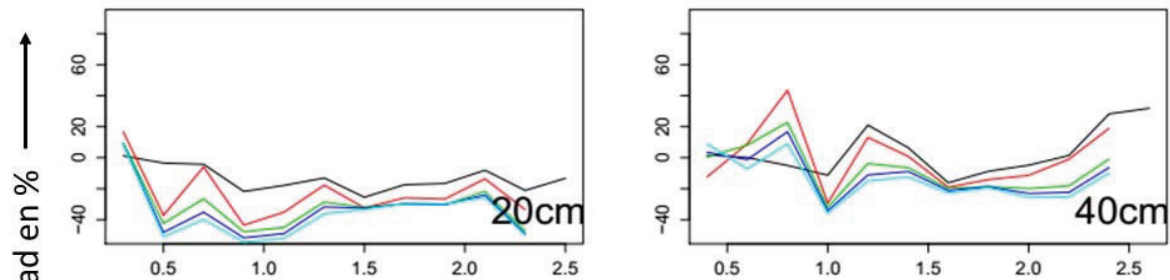

T1

$\mathrm{T} 2$

T4
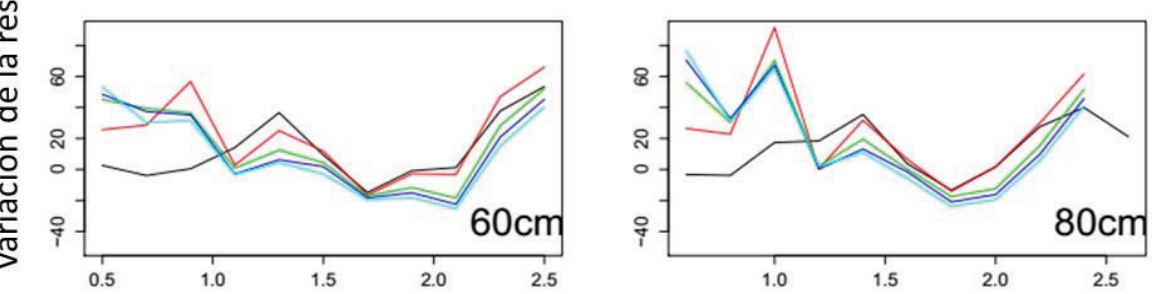

Distancia a lo largo del perfil (m)

Fig. 5b. Variación de la resistividad eléctrica (\%) en diferentes tiempos, a lo largo del perfil, a distintas profundidades en el sitio S2.

Fig. 5 b. Variation of electrical resistivity (\%) at different time periods along the profile, at different depths in site S2.

$\mathrm{T} 1=20 \mathrm{~min}, \mathrm{~T} 2=50 \mathrm{~min}, \mathrm{~T} 3=95 \mathrm{~min}, \mathrm{~T} 4=123 \mathrm{~min}$ 


\section{RECONOCIMIENTO}

El presente trabajo fue financiado por la Universidad de Buenos Aires, Proyecto UBACyT, 00578 .

\section{LITERATURA CITADA}

Arora, T., and S. Ahmed. 2011. Characterization of recharge through complex vadose zone of a granitic aquifer by time-lapse electrical resistivity tomography. J. Appl. Geophys. 73:35-44.

Auge, M.P. 2004. Regiones Hidrogeológicas. República Argentina y Provincias de Buenos Aires, Mendoza, Santa Fe. Facultad de Ciencias Exactas y Naturales. Universidad de Buenos Aires. Disponible en http://sedici. unlp.edu.ar/handle/10915/15909 (Consulta 15 de agosto 2018).

De Franco, R.G., L. Biella, P. Tosi, A. Teatini, B. Losej, A. Chiozzotto, et al.. 2009. Monitoring the saltwater intrusion by time lapse electrical resistivity tomography: the Chioggia test site (Venice lagoon, Italy). J. Appl. Geophys. 69:117-130.

Denoia, J., O. Sosa, G. Zerpa, B. Martín. 2000. Efecto del pisoteo animal sobre la velocidad de infiltración y sobre otras propiedades físicas del suelo. Rev. Pastos 30(1):129-141.

Domínguez, M., A. Jurado, y M.J. Polo. 2011. Influencia del tiempo de encharcamiento en la transferencia de solutos del suelo al flujo de escorrentía. Estudios en la Zona No Saturada del Suelo 10:229-234. Disponible en www. zonanosaturada.com/zns11/publications/ p229.pdf.

García A.R., S.N. Fleitte, I. Ciapparelli, D. Vázquez Pugliese, C. Weigandt, A. Fabrizio de Iorio. 2015. Observaciones, desafíos y oportunidades en el manejo de efluentes de feedlot en la provincia de Buenos Aires, Argentina. Asociación Argentina de Ecología. Ecología Austral 25:255-262.

García A.R., S.N. Fleitte, D. Vázquez Pugliese, and A. Fabrizio de Iorio . 2013. Feedlots and pollution-A growing threat to water resources of agro-production zone in Argentina. Environ. Sci. Tech. 47(21):1193211933.

Garel E., V. Marc, S. Ruy, A.-L Cognard-Plancq, S. Klotz, C. Emblanch, et al. 2012. Large scale rainfall simulation to investigate infiltration processes in a small landslide under dry initial conditions: the Draix hillslope experiment. Hydrolog. Proces. 26(14):21712186 DOI: 10.1002/hyp.9273
Farrakh Nawaz, M., G. Bourrié, and F. Trolard. 2013. Soil compaction impact and modelling. A review. Agron. Sustain. Dev. 33:291-309. DOI 10.1007/s13593-011-0071-8

Fernández, P.L., C.R. Alvarez., and M.A. Taboada. 2015. Topsoil compaction and recovery in integrated no-tilled crop-livestock systems of Argentina. Soil and Tillage Research 153:86-94.

Flores, H., J.L. Arumi, D. Y. Rivera, y L.O. Lagos. 2012 A simple method to identify áreas of environmental risk due to manure application. Environ. Monit. Assess. 184(6):3915-3928.

Losinno, B.N., C.M. Sainato., H.J., Malleville, y G. Galindo. 2008. Tomografía de resistividad eléctrica aplicada a la caracterización de sitios contaminados en tambos. Revista Cienc. Suelo 26(2):141-152.

Martínez, D., A. Landini, E. Soza, O.S. Heredia, y C. Sainato. 2010a. Efecto del pisoteo animal sobre las propiedades de un suelo. Parte I. Densidad aparente, humedad, resistencia a la penetración y modelos asociados. AgroCiencia, Chil. J. Agric. Anim.Sci. 27(1):5-14

Martínez, D., A. Landini, E. Soza, C. Sainato, y O.S. Heredia. 2010b. Efecto del pisoteo animal sobre las propiedades de un suelo. Parte II: infiltración y conductividad hidráulica saturada. Agro-Ciencia, Chil. J. Agric. Anim. Sci. 27(1):15-27.

Oldenburg, D.W., and Y. Li. 1994. Inversion of induced polarization data. Geophysics 59:1327- 1341.

Pordomingo, A., 2013. Feedlot. Alimentación, diseño y manejo. EEA "Guillermo Covas" INTA Anguil, Facultad de Ciencias Veterinarias, UNLPampa. Boletín técnico $\mathrm{N}^{\circ}$ 95. Agosto 2013. Disponible en https:// inta.gob.ar/sites/default/files/script-tmpinta_feedlot_2013.pdf (Consulta 14 de mayo 2017).

Sainato, C.M., B.N. Losinno, and H.J. Malleville. 2010. Electrical resistivity tomography applied to detect contamination at a dairy farm at the Pampean Region, Argentina. European Association of Geoscientists \& Engineers. Near Surface Geophysics 8(2):163-172.

Sainato, C.M., B.N. Losinno, and H.J. Malleville. 2012. Assessment of contamination by intensive cattle activity through electrical resistivity tomography. J. Appl. Geophys. 76:82-91. DOI:10.1016/jjappgeo.2011.10.010.

Samouelian, A., I. Cousin, A. Tabbagh, A. Bruand, and G. Richard. 2005. Electrical resistivity survey in soil science: a review. Soil \& Tillage Research 83:173-193. 
Telford, W.M., L.P. Geldart, and R.E. Sheriff. 1990. Applied Geophysics. 770 p. $2^{\text {nd }} \cdot$ ed. Cambridge University Press, Cambridge, UK.

Travelletti, J., P. Sailhac, J.P. Malet, G. Grandjean, and J. Ponton. 2012. Hydrological response of weathered clay-shale slopes: water infiltration monitoring with time-lapse electrical resistivity tomography. Hydrolog. Process. 26:2106-2119. DOI: 10.1002/ hyp.7983.

Wehrer, M., and L.D. Slater. 2015. Characterization of water content dynamics and tracer breakthrough by 3-D electrical resistivity tomography (ERT) under transient unsaturated conditions. Water Resour. Res. 51(1):97-124.

Zieher, T., G. Markart, D. Ottowitz, A. Römer, M. Rutzinger, G. Meißl, et al. 2017. Water content dynamics at plot scale-comparison of timelapse electrical resistivity tomography monitoring and pore pressure modelling. J. Hydrol. 544:195-209. 